\title{
Gradient flows: from theory to application
}

\author{
Bertram Düring, Carola-Bibiane Schönlieb and Marie-Therese Wolfram
}

June 20, 2016

\begin{abstract}
This volume contains selected contributions related to the international workshop on Gradient flows: from theory to application, held at the International Centre for Mathematical Sciences in Edinburgh during 20-24 April 2015.
\end{abstract}

Partial differential equations (PDE) have been used successfully to describe a variety of important phenomena in physics, engineering, life and social sciences. Many of these processes are driven by minimising energies with respect to certain costs, following the common rule in nature to be as efficient as possible. Hence a large class of nonlinear PDEs can be interpreted as gradient flows in certain metrics, in which the energy along solutions decreases as fast as possible. The choice of the energy as well as the dissipation mechanism allows for a variety of formulations. The heat equation for example can be interpreted as an $L^{2}$-gradient flow of the $H^{1}$-seminorm $E=\frac{1}{2} \int|\nabla u|^{2}$, but also as a Wasserstein gradient flow of the entropy $E=\int u \log u$. Both interpretations have their merits, although the latter might be considered a more 'natural' measure to describe the state of a system. Wasserstein gradient flows have become a popular tool in PDE analysis, especially since the seminal work of Jordan, Kinderlehrer and Otto, cf. [21]. They demonstrated that solutions of the Fokker-Planck equation can be interpreted as a steepest descent of the entropy functional with respect to the Wasserstein metric. The connection between Wasserstein metrics and dynamic systems involving dissipation or diffusion initiated a lot of research on the analysis of gradient flows, see for example the monograph by Ambrosio, Gigli and Savaré [2]. It allowed for further developments in the field of optimal transportation problems, see $[36,34]$ and set the basis for the development of numerical schemes. Also research on the connection of gradient flow structures to the underlying microscopic particle systems by studying the large deviation behaviour [1] or the GENERIC (General Equation for NonEquilibrium Reversible-Irreversible Coupling) framework to describe, in one 
system, both reversible and irreversible dynamics [19, 30, 27] were influenced by the research on Wasserstein gradient flows.

These recent developments also fertilised research in different fields of applied mathematics. Starting with the development of analytic tools for nonlinear PDEs describing transport phenomena in general, research quickly spread into areas such as medical imaging and image processing as well as applied PDE theory in the life and social sciences. Many well known mathematical models in physics, such as the porous medium equation, see e.g. [35], or the Derrida-Lebowitz-Speer-Spohn equation [14, 17], can be interpreted as gradient flows. In material sciences gradient flow systems have been used to describe the behavior of plastic materials, see [28], or to model phase separations of two materials, e.g. the Cahn-Hilliard equation [10]. The latter also has applications in image processing, such as image restoration $[5,9]$. Gradient flow techniques have been used successfully to analyse transportation processes in biology, for example for the Patlak-Keller-Segel equation [22] modeling cell motion in the presence of a chemoattractant or aggregation models for many-particle systems. Here the decay properties of the energy functionals were used to show existence of solutions and study the convergence behaviour towards complex stationary states, see for example $[12,8]$. In the context of PDE on surfaces as well as surface processing, gradient flows constitute a rich class of models such as the formulation of thin films on surfaces [33, 3], or the gradient flow perspective for formulating a level set representation of Willmore flow [15].

Gradient flows also have a long standing tradition in image processing, in particular for image enhancement, such as classical approaches for total variation minimisation have been formulated as gradient flows [37] and generalisations of this to anisotropic diffusions [38]. It is also more generally connected to questions of variational regularisation techniques in inverse imaging problems, and more recently - in the context of the Wasserstein distance - has been used for image registration, warping, shape classification and image segmentation [20,32, 23].

The gradient flow formalism provides a natural framework to preserve physical properties, such as the positivity of solutions or the entropy. Hence the implementation of numerical schemes received considerable attention in the last years. Starting with the seminal work of Benamou and Brenier [4] who based their solver on the hydrodynamic formulation of classical mass transportation problems, different discretization techniques have been proposed. Most of them are based on the semi-discretisation of the gradient flow equations $[7,11]$ or the Lagrangian interpretation, see for example $[18,13,16,24]$. A common challenge in all these schemes is the compu- 


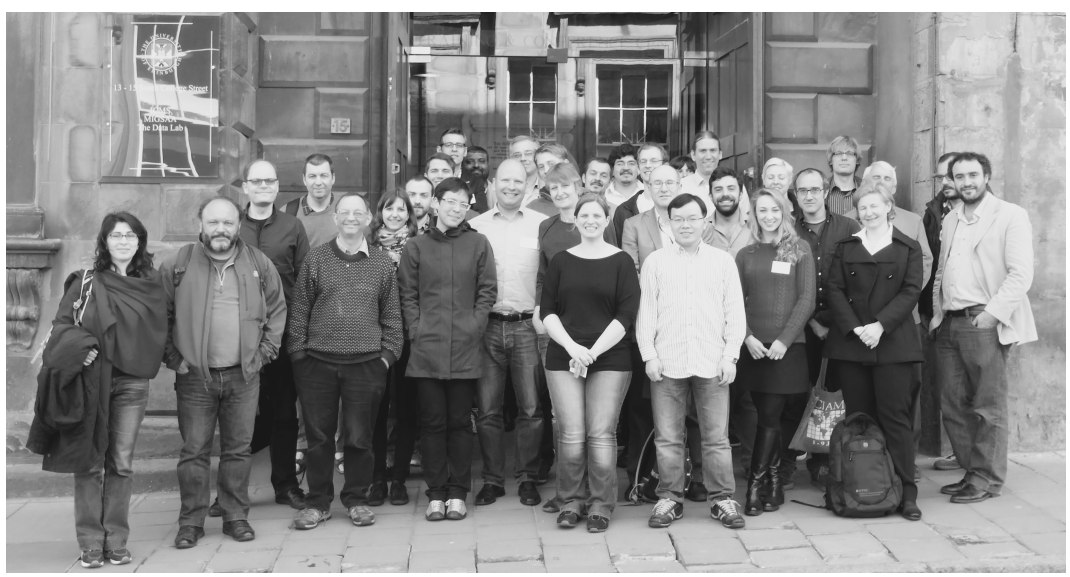

Figure 1: Participants of the ICMS workshop Gradient flows: from theory to application, 22 April 2015.

tational complexity. They either involve the solution of highly nonlinear partial differential equations or involve the computation of the Wasserstein distance, a quantity which corresponds (in space dimension greater than one) to an optimisation problem itself. There are few results on the numerical analysis of these schemes. The first analytic result on the convergence of a Lagrangian scheme in one spatial dimension was provided by Matthes and Osberger in [24]. Recently, when also the imaging community got interested in the Wasserstein distance as a measure of similarity, continuous convex optimisation techniques, such as proximal forward-backward and primaldual iterations, have been proposed for the minimisation of the Wasserstein distance [31]. More generally, dissipation-preserving numerical methods for gradient flows are also developed in geometric integration, such as the discrete gradient method [26, 25].

More recently gradient flow techniques have made its way into practical applications in industry. For example optimal transport distances for full wave form inversion in seismic imaging cf. [29], Hamiltonian flows in weather forecasting cf. [6] and image registration in medicine [20].

This volume was initiated by the International Centre for Mathematical Sciences workshop on 'Gradient flows: from theory to application' held in April 2015. The workshop was attended by 43 participants, both from UK and from overseas. It covered a wide spread of topics in gradient flow research, ranging from analysis and numerics to applications in industry. The contributions to this volume provide a flavour of different areas which 
were discussed at the workshop:

- Benamou, Carlier and Laborde take advantage of the Benamou-Brenier dynamic formulation of optimal transport to propose a variant of the JKO scheme for Wasserstein gradient flows which employs an augmented Lagrangian method in each step of the JKO scheme. They present numerical results for the porous medium equation, nonlocal interaction equations as well as systems of interacting species.

- Di Francesco reviews results from the literature which attempt to link the theory of scalar conservation laws with the Wasserstein gradient flow theory.

- Cesaroni, Dirr and Novaga study a semilinear viscous parabolic equation with periodic nonlinearity, with the aim of characterising the asymptotic speed of propagation of almost planar solutions.

- Bonetti, Rocca, Rossi and Thomas study the well-posedness of equations arising in continuum mechanics when combining plastic deformation and isotropic damage.

We are very grateful to the fantastic staff at ICMS, in particular Ms Audrey Brown, to the members of the advisory board (Andrea Bertozzi, Martin Burger, José A. Carrillo and Peter Markowich) and to all participants.

\section{References}

[1] Stefan Adams, Nicolas Dirr, Mark Peletier, and Johannes Zimmer. Large deviations and gradient flows. Philosophical Transactions of the Royal Society of London A: Mathematical, Physical and Engineering Sciences, 371(2005):20120341, 2013.

[2] L. Ambrosio, N. Gigli, and G. Savaré. Gradient flows. Springer, 2005.

[3] Omri Azencot, Orestis Vantzos, Max Wardetzky, Martin Rumpf, and Mirela Ben-Chen. Functional thin films on surfaces. In Proceedings of the 14th ACM SIGGRAPH/Eurographics Symposium on Computer Animation, pages 137-146. ACM, 2015.

[4] J.-D. Benamou, Y. Brenier, and K. Guittet. The Monge-Kantorovitch mass transfer and its computational fluid mechanics formulation. Internat. J. Numer. Methods Fluids, 40(1-2):21-30, 2002. ICFD Conf. on Num. Methods for Fluid Dynamics (Oxford, 2001). 
[5] Andrea L Bertozzi, Selim Esedoglu, and Alan Gillette. Inpainting of binary images using the Cahn-Hilliard equation. IEEE Transactions on image processing, 16(1):285-291, 2007.

[6] C. J. Budd, M. J. P. Cullen, and E. J. Walsh. Monge-ampére based moving mesh methods for numerical weather prediction, with applications to the eady problem. J. Comput. Phys., 236:247-270, March 2013.

[7] Martin Burger, Jos A. Carrillo, and Marie-Therese Wolfram. A mixed finite element method for nonlinear diffusion equations. Kinetic and Related Models, 3(1):59-83, 2010.

[8] Martin Burger, Razvan Fetecau, and Yanghong Huang. Stationary states and asymptotic behavior of aggregation models with nonlinear local repulsion. SIAM Journal on Applied Dynamical Systems, 13(1):397$424,2014$.

[9] Martin Burger, Lin He, and Carola-Bibiane Schönlieb. Cahn-Hilliard inpainting and a generalization for grayvalue images. SIAM Journal on Imaging Sciences, 2(4):1129-1167, 2009.

[10] John W Cahn and John E Hilliard. Free energy of a nonuniform system. i. interfacial free energy. The Journal of chemical physics, 28(2):258$267,1958$.

[11] José A Carrillo, Alina Chertock, and Yanghong Huang. A finite-volume method for nonlinear nonlocal equations with a gradient flow structure. Communications in Computational Physics, 17(01):233-258, 2015.

[12] José A Carrillo, Marco DiFrancesco, Alessio Figalli, Thomas Laurent, Dejan Slepčev, et al. Global-in-time weak measure solutions and finitetime aggregation for nonlocal interaction equations. Duke Mathematical Journal, 156(2):229-271, 2011.

[13] José A Carrillo and J Salvador Moll. Numerical simulation of diffusive and aggregation phenomena in nonlinear continuity equations by evolving diffeomorphisms. SIAM Journal on Scientific Computing, 31(6):4305-4329, 2009.

[14] Bernard Derrida, Joel L Lebowitz, Eugene R Speer, and Herbert Spohn. Fluctuations of a stationary nonequilibrium interface. Physical review letters, 67(2):165, 1991. 
[15] Marc Droske and Martin Rumpf. A level set formulation for willmore flow. Interfaces and free boundaries, 6(3):361-378, 2004.

[16] Bertram Düring, Daniel Matthes, and Josipa Pina Milišic. A gradient flow scheme for nonlinear fourth order equations. Discrete Contin. Dyn. Syst. Ser. B, 14(3):935-959, 2010.

[17] Ugo Gianazza, Giuseppe Savaré, and Giuseppe Toscani. The wasserstein gradient flow of the fisher information and the quantum driftdiffusion equation. Archive for rational mechanics and analysis, 194(1):133-220, 2009.

[18] Laurent Gosse and Giuseppe Toscani. Lagrangian numerical approximations to one-dimensional convolution-diffusion equations. SIAM Journal on Scientific Computing, 28(4):1203-1227, 2006.

[19] Miroslav Grmela and Hans Christian Öttinger. Dynamics and thermodynamics of complex fluids. i. development of a general formalism. Phys. Rev. E, 56:6620-6632, Dec 1997.

[20] Steven Haker, Lei Zhu, Allen Tannenbaum, and Sigurd Angenent. Optimal mass transport for registration and warping. International Journal of computer vision, 60(3):225-240, 2004.

[21] R. Jordan, D. Kinderlehrer, and F. Otto. The variational formulation of the Fokker-Planck equation. SIAM J. Math. Anal., 29(1):1-17, 1998.

[22] Evelyn F Keller and Lee A Segel. Model for chemotaxis. Journal of theoretical biology, 30(2):225-234, 1971.

[23] Jan Maas, Martin Rumpf, Carola Schönlieb, and Stefan Simon. A generalized model for optimal transport of images including dissipation and density modulation. arXiv preprint arXiv:1504.01988, 2015.

[24] Daniel Matthes and Horst Osberger. Convergence of a variational lagrangian scheme for a nonlinear drift diffusion equation. ESAIM: Mathematical Modelling and Numerical Analysis, 48(03):697-726, 2014.

[25] Robert I McLachlan, GRW Quispel, and Nicolas Robidoux. Unified approach to hamiltonian systems, poisson systems, gradient systems, and systems with lyapunov functions or first integrals. Physical Review Letters, 81(12):2399, 1998. 
[26] Robert I McLachlan, GRW Quispel, and Nicolas Robidoux. Geometric integration using discrete gradients. Philosophical Transactions of the Royal Society of London A: Mathematical, Physical and Engineering Sciences, 357(1754):1021-1045, 1999.

[27] Alexander Mielke. Formulation of thermoelastic dissipative material behavior using generic. Continuum Mechanics and Thermodynamics, 23(3):233-256, 2011.

[28] Alexander Mielke and Tomáš Roubíček. Rate-independent systems. Theory and Application (in preparation), 2015.

[29] L. Mtivier, R. Brossier, Q. Mrigot, E. Oudet, and J. Virieux. Measuring the misfit between seismograms using an optimal transport distance: application to full waveform inversion. Geophysical Journal International, 205(1):345-377, 2016.

[30] Hans Christian Öttinger and Miroslav Grmela. Dynamics and thermodynamics of complex fluids. ii. illustrations of a general formalism. Phys. Rev. E, 56:6633-6655, Dec 1997.

[31] Nicolas Papadakis, Gabriel Peyré, and Edouard Oudet. Optimal transport with proximal splitting. SIAM Journal on Imaging Sciences, $7(1): 212-238,2014$.

[32] Julien Rabin and Nicolas Papadakis. Convex color image segmentation with optimal transport distances. In Scale Space and Variational Methods in Computer Vision, pages 256-269. Springer, 2015.

[33] Martin Rumpf and Orestis Vantzos. Numerical gradient flow discretization of viscous thin films on curved geometries. Mathematical Models and Methods in Applied Sciences, 23(05):917-947, 2013.

[34] F. Santambrogio. Optimal Transport for Applied Mathematicians: Calculus of Variations, PDEs, and Modeling. Progress in Nonlinear Differential Equations and Their Applications. Springer International Publishing, 2015.

[35] J.L. Vazquez. The Porous Medium Equation: Mathematical Theory. Oxford Mathematical Monographs. Clarendon Press, 2007.

[36] C. Villani. Topics in Optimal Transportation. Graduate studies in mathematics. American Mathematical Society, 2003. 
[37] Curtis R Vogel and Mary E Oman. Iterative methods for total variation denoising. SIAM Journal on Scientific Computing, 17(1):227-238, 1996.

[38] Joachim Weickert. Anisotropic diffusion in image processing, volume 1. Teubner Stuttgart, 1998. 